\title{
Prospect of CRISPR/Cas9 technology in sustainable landscape plants
}

\author{
Xiaohui Zhang ${ }^{1}$, Jiang Qiu ${ }^{1, *}$ \\ ${ }^{1}$ College of Ecological Technology and Engineering, Shanghai Institute Of Technology, 201418 Shanghai, China
}

\begin{abstract}
Plant breeding is one of the manifestations of sustainable landscape regeneration. The CRISPR/Cas9 system is a new type of genome-directed editing technology modified from the acquired immune defense response in bacteria and archaea. Due to its efficiency and multi-function, it is widely used in animals, plants, microorganisms and other fields. In sustainable plant landscape, the system makes the gene mutate to improve plant varieties, morphological characteristics, and flowering dates, which can increase the variety of landscape. This article discusses the general situation of CRISPR/Cas9 technology and the progress of plant application, summarizing the problems and countermeasures, and looking forward to the application of sustainable landscape plants. Landscape plants are genetically complex. CRISPR/Cas9 technology can improve plants without genome references and cultivate new ornamental varieties, which can accelerate the breeding process of sustainable landscape plants.
\end{abstract}

\section{Introduction}

Plant diversity is an important manifestation of sustainable landscapes and one of the characteristics of sustainable development of the earth. The plant breeding methods of genome editing technology can mutate and recombine the genetic material of plants to obtain new plant varieties. Due to the high cost, time-consuming, and limitations of traditional breeding methods to obtain good target traits, the breeding of new varieties of good traits from parents and the hybridization of good germplasm resources have the disadvantages of high investment and low efficiency. CRISPR technology has high mutation efficiency which is more convenient, and it has the advantage of editing multiple genes at the same time. So far, it has realized the improvement of plants at the genome level and has developed into one of the most popular ways of plant gene editing.

At present, the mutant phenotype dicot plants obtained by CRISPR technology knockout, site-specific substitution or gene insertion and ornamental are poplar, including white poplar, petunia, chrysanthemum, clover and citrus. Plants of the same family and genus generally have similar traits. For example, grapefruit, Japanese orange, and pomelo of the same genus as citrus can be used as ornamental trees, and atura and hydrangea of the same family as tomatoes can be used as landscape flowers. By constructing plant promoters suitable for monocotyledonous plants and dicotyledonous plants, plant mutants could be obtained and breeding of new landscape varieties will be carried out. With the in-depth research of CRISPR technology in a variety of plants, landscape plant breeding is just around the corner.
CRISPR technology performs gene editing on landscape plants accurately and efficiently, such as improving plant height, flowering period, color and shape of flowers, leaves and fruits, which can not only enhance the ornamental value and visual effects of gardens, but also facilitate the selection of excellent plant varieties. Certainly, it will provide a new way to promote the sustainable development of the landscape.

\section{CRISPR/Cas9 technology}

CRISPR/Cas [1](clustered regularly interspaced short palindromic repeats /CRISPR-associated protein) system is a complex composed of crRNA (CRISPR RNA), Cas9 protein, tracrRNA (transacti-vating crRNA) and RNase III and the corresponding target gene composition.

\section{1 The mechanism of CRISPR/Cas9 technology}

As an important part of CRISPR, Cas9 protein is a powerful and characteristic protein that can not only process crRNA, but also cut foreign nucleic acids. Through the combined action of CRISPR RNA (crRNA), trans-activated crRNA and Cas9 endonuclease, the Cas protein complex is guided to bind to a specific PAM site to cut the DNA double-strand, which can promote the targeted cleavage of foreign DNA [2-3].Transform tracrRNA and crRNA into a sgRNA (short guide RNA) with guiding function (Figure 1).

\footnotetext{
* Corresponding author: qiujiang@sit.edu.cn
} 


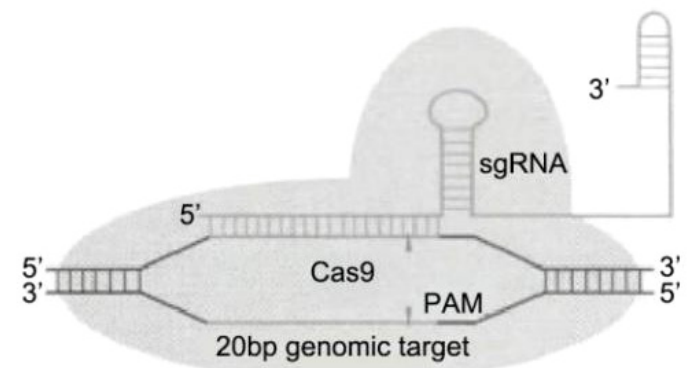

Fig. 1. The mechanism of CRISPR/Cas9 and the structure of sgRNA.

\subsection{The process of CRISPR/Cas9 technology}

The process of CRISPR/Cas9 technology includes primer design, sgRNA expression plasmid construction, target cell transfection, monoclonal isolation and other steps, and finally knock out genes or obtain cell lines with specific point mutations. (Figure 2)

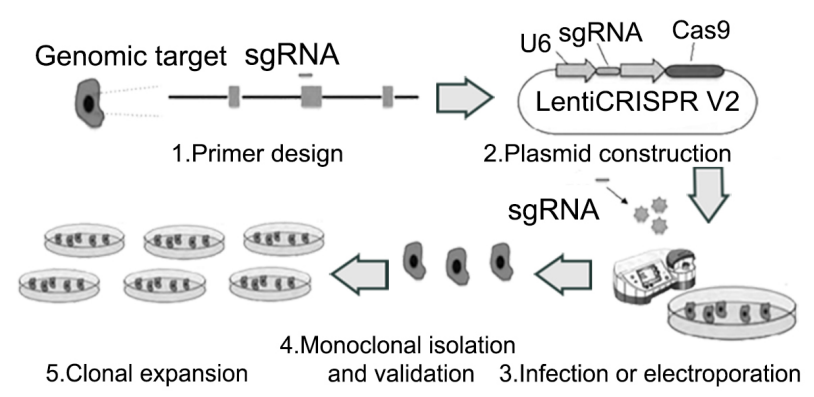

Fig. 2. The basic process of CRISPR/Cas9 technology.

\section{Overview of plant gene research based on CRISPR/Cas9 technology}

In 2013, Ding Wei et al. used CRISPR/Cas9 gene editing technology to link promoters to sgRNA vectors to regulate the transient expression of sgRNA in dicotyledonous plants (soybeans) and monocotyledonous plants (wheat) [4]. Studies proved that the At U6-26 promoter is suitable for dicotyledonous plants, and the Os U6-2 promoter is suitable for monocotyledonous plants. Nowadays, CRISPR/Cas9 technology has been used in gene editing of a variety of plants, such as epigenetic modification, gene expression regulation and plant mutant library.

\subsection{Construction of heritable plant mutants}

In 2013, CRISPR/Cas9 technology was applied to model plants, such as genome editing of Arabidopsis protoplasts, explants, roots, leaf buds and other tissues in order to obtain mutant plants. In 2014, there were researches on constructing promoters to drive human proteins, constructing Arabidopsis promoter-driven sgRNAs vectors, transforming Arabidopsis thaliana, and analyzing its different genetic mutation types and mutation ratios of its three consecutive generations (T1, $\mathrm{T} 2$ and T3), which has verified that the gene mutation induced by CRISPR gene editing technology enables stable inheritance of Arabidopsis [5]. Since 2016, the heritable gene editing of the CRISPR/Cas9 system has covered a variety of diploid plants (rice [6], barley [7], petunia [8], chrysanthemum [9]) and polyploidy plants (canola [10], cotton [11]). In addition, the problems of mutation efficiency, target gene specificity, targeting efficiency, and large fragment deletion were studied.

\subsection{Construction of multi-gene mutants and mutant libraries}

In 2015, CRISPR/Cas9 technology was implemented to target multiple genes and construct mutant libraries. It has proved that CRISPR still has shearing efficiency when sgRNA is mismatched with part of the target gene. By selecting relatively conservative regions or domains in multiple genes or gene families, a single sgRNA can edit multiple target genes at the same time, but the method is limited and conservative. At present, the multi-gene editing method of constructing a tandem transcription cassette between tRNA and sgRNA to process multiple sgRNAs has been widely used in various fields [12]. In 2016, a study achieved rice multigene targeting through the hammerhead nuclease and sgRNA spacer method [13]. In 2017, researchers followed the transcriptome library construction method to screen plants with single-gene mutations, constructed a plant mutant library and applied it to tomato and rice [14].

\subsection{Temporal and spatial specificity of regulating gene expression}

The external environment restricts or promotes plant growth status and gene expression levels. CRISPR/Cas9 technology can edit genes at the DNA level and regulate the transcription level of target genes. DCas9 (dCas9 is mutated from Cas9) that has lost its DNA shearing function can still be accurately identified and combined with the target gene DNA through sgRNA. In 2015, there was research that applied the transcriptional regulation of CRISPR technology to model plant tobacco, which used transient transformation methods to regulate the transcription of protein-coding genes effectively [15]. Studies in the model plant Arabidopsis thaliana proved that CRISPR technology can regulate the transcription of non-protein coding genes by combining the C-terminus of dCas9 with the transcription activation domain VP64 and using sgRNA to target the promoter region of specific genes in order to achieve higher gene expression levels [16].

\section{Prospects}

\subsection{Prospects of CRISPR/Cas9 technology for sustainable landscape plant breeding}

\subsubsection{Gene editing of sustainable landscape plants}


At present, CRISPR/Cas9 technology has been used to achieve multi-gene editing of a variety of crop plants. However, most studies on gene function landscape plant refer to model plants, but not homologous woody plants. Currently, there are few landscape plants involved. For example, woody plants include poplar [17], Populus tomentosa [18] and citrus [19], and herbaceous plants include petunia [20], clover [21] and chrysanthemum [22]. The RNAi method is accurate for targeted silencing of the target RNA of plants theoretically. There are many reference studies on the flowering period and stress research for landscape plants, so it will have broad breeding applicability. Compared with RNAi technology, CRISPR/Cas9 can directly knock out genes and cause loss of gene function efficiently. It is recommended to use RNAi in the exploration stage of landscape plant traits to reduce test costs, and to edit multiple genes simultaneously through CRISPR/Cas9 in the in-depth research stage.

Table 1. Application of CRISPR/Cas9 genome editing technology in genetic improvement of landscape plants

\begin{tabular}{|c|c|c|}
\hline \multicolumn{3}{|c|}{ Dicots } \\
\hline Species & Gene & Editing results \\
\hline $\begin{array}{c}\text { Cabbage(rassica } \\
\text { oleracea L.) }\end{array}$ & Bol,GA4 & Plants dwarf \\
\hline $\begin{array}{l}\text { Poplar(Populus } \\
\text { L.) } \\
\end{array}$ & PtWRKY36 & Not mentioned \\
\hline $\begin{array}{l}\text { Chinese white } \\
\text { poplar( Populus } \\
\text { tomentosa Carr.) }\end{array}$ & $\begin{array}{c}\text { PtPDS1,PtPD } \\
\text { S2 }\end{array}$ & Albinism phenotype \\
\hline $\begin{array}{l}\text { Alfalfa(Medicago } \\
\text { Sativa Linn) }\end{array}$ & hemE & $\begin{array}{c}\text { Promote the } \\
\text { synthesis of vitamin } \\
\text { B12 }\end{array}$ \\
\hline $\begin{array}{l}\text { Chrysanthemum( } \\
\text { Dendranthema } \\
\text { morifolium } \\
\text { (Ramat.) }\end{array}$ & DgGA20ox & Plants dwarf \\
\hline $\begin{array}{c}\text { Tomato(Solanum } \\
\text { lycopersicum) }\end{array}$ & PSY1 & Change fruit color \\
\hline $\begin{array}{l}\text { Tomato(Solanum } \\
\text { lycopersicum) }\end{array}$ & SP5G & $\begin{array}{c}\text { Advance flower } \\
\text { time and increase } \\
\text { fruit yield }\end{array}$ \\
\hline $\begin{array}{l}\text { Sweet orange } \\
\text { (Citrus } \\
\text { sinensis (L.) } \\
\text { Osbeck) }\end{array}$ & CsPDS & Albinism phenotype \\
\hline $\begin{array}{c}\text { Citrus(Citrus } \\
\text { reticulata Blanco) }\end{array}$ & CsLOB1 & $\begin{array}{c}\text { Canker resistant } \\
\text { improvement }\end{array}$ \\
\hline $\begin{array}{c}\text { Cotton(Gossypiu } \\
\text { m spp) }\end{array}$ & ZmABP9 & $\begin{array}{l}\text { Salt tolerance and } \\
\text { drought tolerance }\end{array}$ \\
\hline
\end{tabular}

\subsubsection{Breeding and improvement of sustainable landscape plants}

By changing the ornamental points of plants, such as improving leaf color, plant height, flowering period, fruit color, flower color and stress research. Flowering period regulation is of great significance to the configuration of landscape plants and variety selection. Traditional hybrid breeding has a long cycle, which is complex and inefficient. Knocking out negative regulatory genes that play an important role in inhibiting flower formation by
CRISPR can advance or prolong the flowering period, because early or prolonged flowering or even continuous flowering is the most ideal trait of landscape plants. In 2012, it was proved that the TFL1 gene can regulate the continuous flowering of rose and strawberry [23], and knocking out of the SP5G gene can advance the flowering period of tomatoes [24]. CRISPR/Cas9 technology is a new way to improve new varieties of landscape flowers by knocking out related regulatory genes and regulating the length of flowering.

\subsubsection{Improve the safety of transgenic plants}

Traditional transgenes allow recipient plants to obtain new specific traits through interspecific hybridization, distant hybridization and somatic mutation. Random insertion of foreign genes has hidden dangers such as endogenous gene mutation and gene silencing. The use of controversial marker genes such as antibiotics and herbicide resistance genes may not guarantee biological safety that may cause environmental release, which is risky. CRISPR technology modifies endogenous genes directly to obtain mutants that will not introduce any foreign genes, which can be safely applied to gene mutation and transcriptional regulation of landscape plants.

\section{5 conclusion}

The traditional gene editing technology of epigenetic regulation and plant variety improvement mainly involves random insertion of exogenous genes into the plant genome, including gene gun method, RNAi method, T-DNA insertion method, and pollen pipeline method. Due to its randomness, the damage to host genes is unpredictable, causing problems such as damage to endogenous genes and silencing of exogenous genes. Moreover, the probability of plant genome homologous recombination and genetic mutation of the target trait is reduced. CRISPR can take advantage of its system to increase the probability of homologous recombination and non-terminal homologous recombination of genes, and promote genome mutations accurately and efficiently.

Sustainable landscape is a new and operable method to achieve a sustainable environment and the earth, and it is also a new perspective to face the global crisis. Compared with traditional gene editing technology, CRISPR has strong operability because of its simple principle, low cost, high mutation efficiency and wide applicability. CRISPR stands out in the discovery and verification of plant targets. The mutation of the exons that target the functional domain of the protein is screened for key proteins or domains that maintain specific plant traits, which facilitates efficient acquisition of plants with corresponding traits. CRISPR obtains plant materials without genetically modified fragments through passage, which will facilitate rapid and accurate high-quality variety improvement. 


\section{References}

1. H. Philippe, B. Rodolphe, Science, 327, 5962 (2010)

2. D. Elitza, C. Krzysztof, M.S. Cynthia, G. Karine, C. Yanjie, A.P. Zaid, R.E. Maria, V. Joerg, C. Emmanuelle, Nature, 471, 7340 (2011)

3. G. Giedrius, B. Rodolphe, H. Philippe, V. Siksnys, Proc Natl Acad Sci USA, 109, 39 (2012)

4. Z. Feng, B. Zhang, W. Ding, L. Xiaodong, Y. DongLei, w. Pengliang, C. Fengqiu, Z. Shihua, Z. Feng, M. Yanfei, Z. Jian-Kang, Cell Res, 23, 10 (2013)

5. Z. Feng, Y. Mao, N. Xu, et al., Proc Natl Acad Sci USA, 111, 12 (2014)

6. F. WANG, C. WANG, P. LIU, et al., PLoS ONE, 11, 4 (2016)

7. Y. Lu, X. Ye, R. Guo, et al., Molecular Plant, 10, 9 (2017)

8. H. Wang, Southwest University (2019)

9. C. Li, F.S. Hao, L.R. Sun, Journal of Henan University of Science and Technology (Natural Science Edition), 05 (2018)

10. X.W. Gao, A.Q. Tan, X.C. Hu, et al., Journal of Plant Genetic Resources, 05 (2020)

11. C.L. Wang, China Agricultural University (2017)

12. K. Xie, B. Minkenberg, Y. Yang, Proc Natl Acad Sci USA, 112, 11 (2015)

13. X. Tang, X. Zheng, Y. Qi, et al., Mol Plant, 9, 7 (2016)

14. X. Meng, H. Yu, Y. Zhang, et al., Mol Plant, 10, 9 (2017)

15. Piatek A, Ali Z, Baazim H, et al., Plant Biotechnol, 13, 4 (2015)

16. L.G. Lowder, D. Zhang, N.J. Baltes, et al., Plant Physiol, 169, 2 (2015)

17. Y. Wang, Anhui Agricultural University (2018)

18. T.T Liu, D. Fan, L.Y. Ran, Y. Ling, et al., Inheritance, 10 (2015)

19. H. JIA, Y. ZHANG, V. ORBOVIC, et al., 15, 7 (2017)

20. K. Wang, Southwest University (2019)

21. X.Y. Wu, H.N. Dong, Z.Q. Liu, et al., 06 (2019)

22. C. Li, F.S. Hao, L.R. Sun, Journal of Henan University of Science and Technology (Natural Science Edition), 05 (2018)

23. M. Randoux, J. Jeauffre, T. Thouroude, F. Vasseur, L. Hamama, M. Juchaux, S. Sakr, F. Foucher Fabrice, Journal of Experimental Botany (2012)

24. S. SOYK, N.A. MULLER, S.J. PARK, et al., Nature Genetics, 49, 1 (2017) 